\title{
AN OVERVIEW OF LAN/WLAN IN CAD/CAM/CAE APPLICATIONS
}

\author{
Haswendra A.R. ${ }^{1,2}$ \\ ${ }^{1}$ Lecturer, Kumon Talang Banjar, Jambi, Indonesia \\ ${ }^{2}$ Mechanical Engineering Department, Sriwijaya University, South Sumatera, Indonesia
}

\begin{abstract}
Computer network is an interface of two or more computers that is built for the purpose of sharing, information, data, and resources. Computer network has nodes that serve as the origin, route, and receiver of data. Computer network is built using a combination of hardware and software. LANs and WLANs are the most common form of computer network and they are used intensively and extensively in CAD/CAM/CAE applications. This review talks about the latest implementation of LANs/WLANs in CAD/CAM/CAE applications, their limitations, and consideration for their usage. There are four aspects that a LAN/WLAN system must fulfill which are Fast, Expandable, Reliable, and Secure. LAN and WLAN including their many standards each has different properties which influence their implementation in CAD/CAM/CAE applications.
\end{abstract}

Keywords: LAN, WLAN, CAD, CAM, CAE, Ethernet, Security, Network

\section{INTRODUCTION}

Computer network is an interface of two or more computers that is built for the purpose of sharing, information, data, and resources. Computer network has nodes that serve as the origin, route, and receiver of data. Computer network is built using a combination of hardware and software. Data transmission between network nodes is conducted through various forms of data links like physical cable, Wi-Fi, or even optical communication. There are many ways to categorize computer networks, among them is by using the area it spans. Local Area Networks (LAN) typically span an office, a school, a home, etc. Wide Area Networks (WAN) can connect to a much farther place and they may span an entire city, province, or even the entire world, which is also called the Internet. The limited nature of LANs usually makes the network stay outside the regulations implemented by authorities while the larger coverage of WANs almost always requires the use of public facilities like telephone cables and ISDN, making WANs got supervised more. This makes the implementation of LANs and WANs different despite their similar purpose, to transfer information. In engineering, the importance of transferring information has grown enormously due to the advent of computers and computer numerical control (CNC) which are used extensively and intensively in the various computer-aided technologies including Computer-Aided Drawing (CAD), ComputerAided Manufacturing (CAM), and ComputerAided Engineering (CAE). The popularity of researches that explore the use of computer network to optimize the information used by CAD/CAM/CAE process to improve overall performance of a system shows that information has already been considered an important production factor [1].

Ethernet is virtually the only wired local area network technology still in use nowadays. Other technologies exist, but they either have market share so small that no one ever heard of them, or they are technologies used only by specific organizations. Nowadays, Ethernet is akin to standard plumbing that came with every new compute because of so ubiquitous it is [2]. Ethernet's popularity stems from its ease of configuration, speed, and simplicity. During its evolution, Ethernet has been improved in many different aspects including its bandwidth, medium access control methods, and physical media. The Ethernet has many standard over the years, with the current standards of Ethernet being the IEEE 802.3bz standard for 2.5 Gbps Ethernet and IEEE 5GBASE-T for 5 Gbps Ethernet. 
Computer network is not limited only to wired systems. Wi-Fi is a wireless local area network (WLAN) technology that provide much more convenience. This convenience has made Wi-Fi so widespread and important to daily lives of people. Wi-Fi enables multiple devices within Wi-Fi's coverage range to connect wirelessly to its "hotspot". Wi-Fi has an undeniable advantage over Ethernet in which it needs no cables to connect to multiple devices, enabling its use to control cordless robots which need to move around while constantly receiving control orders. This is often seen in logistics application where tens, hundreds, or even more robots are utilized to move packages around [3][4]. Wi-Fi coverage is limited to around $30 \mathrm{~m}$ indoors and $100 \mathrm{~m}$ outdoors, but various aspects like walls and signal interference may influence its range and also its error bit rate [5]. Wi-Fi has some protocols that are commonly in use nowadays including IEEE $802.11 \mathrm{~g}$ with data rate of $54 \mathrm{Mbps}$, IEEE 802.11n for data rate of $150 \mathrm{Mbps}$, and the latest IEEE 802.11ac for a high data rate of 866.7 Mbps.

In the context of CAD/CAM/CAE, information can include various kinds of data. This includes images, drawings, and specifications needed for documentation of projects and systems the company runs, control programs to control NC machines and robots, log of actions and breakdowns to be used to optimize future operations, and data concerning inventory management and ordering. Proper management and transfer of information is critical in improving a company's performance and profit, which serves as an incentive for the greater interests companies have in data networking [6]. This paper reviews the many different desired properties of LAN/WLAN in CAD/CAM/CAE applications and how well can they fulfill those.

\section{DESIRED LAN PROPERTIES}

To efficiently manage and transfer information, the use of computers, databases, proper software along with installing an information network is essential. The information network must be configured in such a way that all users can easily and immediately access all information that they need. The data network that handle the information processed by a CAD/CAM/CAE company must fulfill certain aspects which are [7]:

\subsection{Fast}

LANs/WLANs that offer good performance at first may become inadequate after some time because of various bottlenecks. It is critical for LANs/WLANs to work fast without creating any bottleneck in the network system. LAN congestion is rarely found until recently. LAN used to be able to send more data over time than computers can output. Moreover, the number of users and traffic that use the Internet, which of course uses LANs to deliver its data to users, have increased significantly compared to the Internet's early days, where the military was the sole user of it. The advent of computation, communication, and the bigger reliance on networking and Internet of things seen today have shifted the bottleneck of information processing to the LANs/WLANs [8]. Some factors that can be used to determine whether LANs/WLANs need a speed upgrade:

Higher network utilization: Channel utilization can be measured by the percentage of time a communication path is busy carrying data. The increase of traffic loads translate to higher network utilization. Certain LANs/WLANs hubs and switches can provide their network utilizations. It is also possible to use protocol analyzers or remote monitoring to get a grasp of the network utilizations. Peak network utilization means that the network has become bottleneck, choking the data transfer speed [9].

Increased Collision Counts: A collision happens when multiple stations in a shared network send Ethernet packages at the same time because they sense a quiet network. When this happens, the data becomes corrupted and the stations must retransmit the data. The stations must wait a random amount of time before they send their packets again. This reduces the overall throughput of a network system [10]. The increase in the number of packet collisions usually means higher network congestion.

Degradation of Application Performance: In a congested network, applications cannot perform to their fullest potential. Data transfer may be slow and session time out may happen more often. This degradation can also be caused by other factors, but this can serve as one of the markers of a congested network [6].

Increased Network Delay: All LANs/WLANs have a rated maximum speed. When data transfer surpassed that limit and the LANs/WLANs were overloaded, the transfer may need unusually more 
time to be completed. This results in the increase of average delay or service time, making the network seems slow to its users. In $\mathrm{CAD} / \mathrm{CAM} / \mathrm{CAE}$ application, the ongoing trend is of multiple users working together on a project at the same time, in real-time, where a change made by a single user is immediately shown to the other users so that they can respond accordingly [11][12]. This is intended to smoothen out the work process of a team project, erasing the needless time spent on sharing and synchronizing work progress manually. Network delay would hamper this process. For this working process to be conducted smoothly, synchronization or the preservation of temporal dependencies of the media between the users must occur as quickly as possible [13].

Bottleneck due to Increasing Storage Speed: With the mass adoption of Solid-State Drive (SSD) and NVMEs in enterprise segment, the need for faster LANs/WLANs materializes. For example, when using older standards of WLANs, modern storage speed nowadays vastly outpaces the transfer speed through the network, creating a bottleneck. When the storage is set in RAID-0 configuration which processes requests in bulks, the bottleneck becomes even more noticeable, showing up as significant bandwidth fluctuations between zero and peak usage [9]. This creates an imbalance of the performance in the system. An upgrade that is going to be applied to a network system must be considered thoroughly. The entire network must receive proper upgrade to avoid bottlenecks that may reduce efficiency of network system. This is to avoid unnecessary waste of budget.

\subsection{Expandable}

The data network built must also leave some room for future expansion of the system to better handle the growth of the company and the increase of the amount of information processed. As the CAD/CAM/CAE company grows, the network must expand to include new employees, workgroups, systems, equipment, suppliers and customers. The possibility for expansion must be considered beforehand to make network expansion easier to accomplish.

The network must be able to accommodate newer personal computer interface (PCI) and novel data storage systems used in many new computers. The latest developments in Solid-State Drive (SSD) technology has cornered the traditionally-used Hard Disk Drive (HDD) in the enterprise segment. SSDs have significant advantages in various key points including average access time, input operations per second, and sequential read and write speed. Enterprise class SSDs have reached sequential read speed of $500 \mathrm{Mb} / \mathrm{s}$ and write speed of $250 \mathrm{Mb} / \mathrm{s}$ while enterprise class HDDs only reach $200 \mathrm{Mb} / \mathrm{s}$ read and write speed. This implementation saves hours of access time per computer [14]. The ongoing improvements on storage speed soon make old LANs/WLANs standards inadequate. In this case, an upgrade to network bandwidth must be conducted to keep up with the efficiency provided by other parts of the system.

\subsection{Reliable}

Reliability means that the LANs/WLANs systems that CAD/CAM/CAE company use must be dependable. The network must able to provide good Quality of Service for its users. Quality of Service is determined from network throughput and delay. There is a fundamental reliability difference between LANs and WLANs that may affect decision to use one system over another for $\mathrm{CAD} / \mathrm{CAM} / \mathrm{CAE}$ applications. Ethernet LAN has the best channel efficiency of $100 \%$ while WLAN has lower channel efficiency percentage. With the increase of distance, WLANs see noticeable increase in delay while LANs are mostly unaffected[5].

\subsection{Secure}

LANs/WLANs for CAD/CAM/CAD company must be protected from outside threat. Projects conducted by a CAD/CAM/CAD company may range from military weapons to the emerging research of self-driving automotive [15] [16] [17] to the logistics industry. All of which include a possibility of huge financial losses or even death if the project is leaked, manipulated, or destroyed.

The motives for the attacker of LANs/WLANs networks can be various, some of them are: (1) Learning about the network in question to ease his way when he attempts to deal more damage later on, (2) eavesdropping and stealing data, (3) information manipulation and sabotage, (4) disrupting the network in various forms [18]

LANs and WLANs have different security properties. WLANs are generally less safe because the aim of its inception is to conveniently create a networking environment with little to no security. The success of WLANs and their fast adoption, especially in the area where security is of necessary. This created a problem that 
WLAN's pioneers did not consider during its inception [19]. New WLAN protocols have been released to minimize the issue, but WLANs are still generally unsafe compared to LANs especially due to its own advantage of being accessible from anywhere within its transmission area, not needing any cables or physical access to connect. The confidentiality of the data transmitted though WLAN network can be compromised comfortably from outside of its owner's premise [20].

\section{CONCLUSIONS}

The use of LANs/WLANs for CAD/CAM/CAE optimization is on the rise. Each has its own advantages and many more to be discovered. LANs have the advantage in speed, security, and reliability over long distances over WLANs while WLANs is considered very practical and convenient for low-security applications. An upgrade to the network system of a $\mathrm{CAD} / \mathrm{CAM} / \mathrm{CAE}$ company is recommended if the network system came short in one of the desired LAN/WLAN properties mentioned. But an improper upgrade may introduce bottlenecks which may reduce the efficiency of the newly upgraded parts, therefore careful consideration before an upgrade is important. Network system is a fast-developing field in which significant innovations and improvements are made each year. The implementation and upgrade of LAN/WLAN for CAD/CAM/CAE company must always consider both the latest and greatest of the systems and also proven technologies.

\section{REFERENCES}

[1] S. A. S. Obayes, I. R. K. Al-Saedi, and F. M. Mohammed, "Prototype Wireless Controller System Based on Raspberry Pi and Arduino for Engraving Machine," Proc. - 2017 UKSim-AMSS 19th Int. Conf. Model. Simulation, UKSim 2017, pp. 69-74, 2018, doi: 10.1109/UKSim.2017.20.

[2] B. Metcalfe, "Metcalfe's Law after 40 Years of Ethernet," Computer (Long. Beach. Calif)., vol. 46, no. 12, pp. 26-31, 2013, doi: 10.1109/MC.2013.374.

[3] A. Catarina and P. Pinto, "Advanced Mobile Manipulation for Logistics in Hospitals or Laboratories," 2016.

[4] Q. P. Ha, L. Yen, and C. Balaguer, "Robotic Autonomous Systems for Earthmoving in Military Applications," Autom. Constr., vol.
107, no. August, 2019, doi: 10.1016/j.autcon.2019.102934.

[5] I. S. A. Abdullah, A. Babiker, and A. N. Mustafa, "The Impact of Distance on WLAN and LAN Network Performance," Int. J. Sci. Res., vol. 5, no. 2, pp. 1063-1065, 2016, doi: 10.21275/v5i2.nov161252.

[6] A. Naz, A. U. Qureshi, and S. Rai, "IMPROVING LAN PERFORMANCE \& SPEED BY FAST ETHERNET TECHNOLOGY," 2017.

[7] H. B. Kief and H. A. Roschiwal, "CNC Handbook," McGraw-Hill Education, 2012, p. 390.

[8] A. F. H. Leon, O. J. S. Parra, and M. J. E. Rico, "Congestion Control in Lan-to-Lan Connections," Internet Distrib. Comput. Syst., no. 10th International Conference, pp. 142-151, 2017.

[9] B. Kim, J. Kim, and S. H. Noh, "Managing Array of SSDs When the Storage Device Is No Longer the Performance Bottleneck," 9th USENIX Work. Hot Top. Storage File Syst. HotStorage 2017, co-located with USENIX ATC 2017, 2017.

[10] A. M. Khalaf, M. S. A. El Salam, and K. A. Ahmed, "Investigation of Different Ethernet Wiring and Different Frame Size to Enhance the Performance of LAN," Int. Conf. Adv. Commun. Technol. ICACT, vol. 5, no. 2, pp. 787-796, 2017, doi: 10.23919/ICACT.2017.7890228.

[11] X. Lv, F. He, Y. Cheng, and Y. Wu, "A Novel CRDT-Based Synchronization Method for Real-Time Collaborative CAD Systems," Adv. Eng. Informatics, vol. 38, no. July, pp. 381-391, 2018, doi: 10.1016/j.aei.2018.08.008.

[12] Y. Wu, F. He, and S. Han, "Collaborative CAD Synchronization Based on a Symmetric and Consistent Modeling Procedure," Symmetry (Basel)., vol. 9, no. 4, pp. 1-20, 2017, doi: 10.3390/sym9040059.

[13] M. Mu, H. Stokking, and F. den Hartog, Network Delay and Bandwidth Estimation for Cross-Device Synchronized Media. 2018.

[14] R. M. A. M. K. Eshghi, "Inside Solid State Drives," vol. 37, pp. 203-231, 2013, doi: 10.1007/978-94-007-5146-0. 
[15] C. W. Lin and H. Yu, "Invited - Cooperation or Competition?: Coexistence of Safety and Security in next-Generation Ethernet-Based Automotive Networks," Proc. - Des. Autom. Conf., vol. 05-09-June, 2016, doi: 10.1145/2897937.2905006.

[16] B. B. Kraemer and I. S. Association, "Commentary Automotive Ethernet," no. December, p. 2016, 2016.

[17] C. Corbett, E. Schoch, F. Kargl, and P. Felix, "Automotive Ethernet: Security Opportunity or Challenge?," Lect. Notes Informatics, pp. 45-54, 2016.
[18] T. Kiravuo, M. Sarela, and J. Manner, "A Survey of Ethernet LAN Security," IEEE Commun. Surv. Tutorials, vol. 15, no. 3, pp. 1477-1491, 2013, doi: 10.1109/SURV.2012.121112.00190.

[19] A. M. Al Naamany, A. Al Shidhani, and H. Bourdoucen, "IEEE 802 . 11 Wireless LAN Security Overview," Ijcsns, vol. 6, no. 5, pp. 138-156, 2006.

[20] P. Feng, "Wireless LAN Security Issues and Solutions," Proc. - 2012 IEEE Symp. Robot. Appl. ISRA 2012, pp. 921-924, 2012, doi: 10.1109/ISRA.2012.6219343. 